\title{
Blood Pressure Intervention Levels In Preterm Infants: Pilot Randomised Trial
}

Sujith S. Pereira MRCPCH ${ }^{1,2}$, Ajay K. Sinha FRCPCH ${ }^{1,2}$, Joan K. Morris $\mathrm{PhD}^{3}$, David F. Wertheim $\mathrm{PhD}^{4}$, Divyen K. Shah $\mathrm{PhD}^{1,2}$, Stephen T. Kempley FRCPCH ${ }^{1,2}$

${ }^{1}$ Neonatal Unit, Royal London Hospital, Barts Health NHS Trust, London, UK

${ }^{2}$ Centre for Genomics and Child Health, Blizard Institute, Barts and the London School of Medicine, London, UK

${ }^{3}$ Wolfson Institute of Preventive Medicine, Queen Mary University of London, London, UK

${ }^{4}$ Faculty of Science, Engineering and Computing, Kingston University, Surrey, UK

Correspondence to: Dr. Steve Kempley, Centre for Genomics and Child Health, Blizard Institute, Barts and The London School of Medicine and Dentistry, 4, Newark Street, London, E1 2AT Tel: +44 (0) 2078822620

s.t.kempley@qmul.ac.uk

Key words: Blood pressure, extremely preterm infant, randomised trial

Trial Registration: ISRCTN83507686 https://www.isrctn.com/ISRCTN83507686 


\section{ABSTRACT}

Objective: To examine the feasibility of a trial allocating different blood pressure (BP) intervention levels for treatment in extremely preterm infants.

Design: Three-arm open randomised controlled trial performed between February 2013, and April 2015.

Setting: Single tertiary level neonatal intensive care unit.

Patients: Infants born $<29$ weeks gestation were eligible to participate, if parents consented and they did not have a major congenital malformation.

Interventions: Infants were randomised to different levels of mean arterial BP at which they received cardiovascular support: Active $(<30 \mathrm{mmHg})$, Moderate ( $<$ Gestational age mmHg) or Permissive (signs of poor perfusion or $<19 \mathrm{mmHg}$ ). Once this threshold was breached, all were managed using the same treatment guideline. BP profiles were downloaded continuously; cardiac output and carotid blood flow were measured at 1and 3 days, amplitude integrated EEG was recorded during the first week. Cranial ultrasound scans were reviewed blind to study allocation.

Main outcome measure: Inotrope usage and achieved BP.

Results: Of 134 cases screened, 60 were enrolled, with mean gestation 25.8 weeks (SD 1.5) and birth weight 817g (SD 190). Invasively measured BP on the first day, and inotrope usage, were highest in the Active and lowest in the Permissive arms. There were no differences in hemodynamic or EEG variables, or in clinical complications. Predefined cranial ultrasound findings did not differ significantly; no infants in the Active arm had parenchymal brain lesions.

Conclusion: The BP threshold used to trigger treatment affects the achieved BP and inotrope usage, and it was possible to explore these effects using this study design. 


\section{Contributors}

SSP, AKS, DKS and STK devised the study concept. SSP, AKS, DKS, DFW, JKM and STK developed the study protocol and designed the study. SSP and STK supervised and gathered data. SSP, STK analysed and interpreted the data. DFW analysed the electroencephalographic data. JKM performed statistical analysis. SSP and STK drafted the report. SSP, AKS, DFW, JKM, DKS and STK critically revised the report. SSP and STK had full access to the data and take responsibility for the integrity of the data and accuracy of the analysis. 


\section{INTRODUCTION}

The management of cardiovascular disturbances in extremely preterm infants differs considerably between neonatal units. ${ }^{1-6}$ Blood pressure (BP) is routinely measured, some studies show low BP on the first day is associated with adverse outcomes, ${ }^{7}$ but observational studies have not shown links with neurodevelopmental sequelae. ${ }^{89}$ The criteria for supporting low BP has been identified as a research priority. ${ }^{10}$

With challenges in estimating cardiac output and systemic vascular resistance, BP is frequently used as a proxy. ${ }^{11-13}$ The 'normal' range of BP for extremely preterm infants remains elusive and whether such ranges ensure optimal organ perfusion remains unknown. ${ }^{214}$

Over two decades ago a pragmatic approach was proposed to maintain mean arterial BP (MABP in mmHg) above the gestation of the infant in weeks ${ }^{15}$ and this remains widely practiced. ${ }^{4516}$ Some units practice permissive hypotension whereby BP is not supported without clinical or biochemical evidence of poor perfusion. A minority of units maintain MABP above $30 \mathrm{mmHg}^{16}$, with some studies showing cerebral white matter changes ${ }^{17}$ or impaired autoregulation ${ }^{18}$ with BP below this level.

Observational studies have suggested an association between treatment for hypotension and adverse outcomes. ${ }^{19}$ Anti-hypotensive treatment may simply identify cardiovascular instability, but could itself be harmful. Randomised trials need to examine whether low BP in extremely preterm infants should be supported, to assess whether inotropic agents are beneficial or harmful, and to determine BP levels at which clinicians should intervene. 
The aim of this study was to compare in extremely preterm infants ( $\leq 28$ weeks), different BP intervention levels at which clinicians should commence circulatory support in the neonatal unit (these intervention levels were MABP $<30 \mathrm{mmHg}$, MABP <gestation, or treating impaired perfusion and/or MABP $<19 \mathrm{mmHg}$ ). We hypothesised that a higher BP intervention level would represent a lower threshold for treatment, resulting in higher achieved BP during the first week of life, and more inotropic agent usage during the infant's neonatal unit admission. 


\section{METHODS}

\section{Study design}

This 3-arm parallel pilot single centre randomised clinical trial investigated intervention levels for BP support in extremely premature neonates. It was conducted in a tertiary medical and surgical neonatal intensive care unit (NICU) with 36 cots and over 600 annual admissions.

\section{Participants}

Infants were eligible if born $<29$ weeks gestation, recruited and randomised within 12 postnatal hours. The only exclusions were major congenital malformation, lack of parental consent, or lack of assent from clinicians. Written information was provided to parents when delivery was anticipated, and parents offered assent before delivery. Written consent was obtained within 12-hours following delivery. For unexpected deliveries or postnatal transfers, this took place after admission. Postnatal consent was required by the ethics committee, following a parent consultation in which $67 \%$ stated a preference for formal postnatal consent before recruitment.

\section{Randomisation and masking}

Randomisation was performed using stratification in individual weeks of gestation, with permuted blocks of 3 patients. A random number generator allocated blocks, with allocation concealed in sequentially numbered opaque sealed envelopes (by STK). Clinicians were unaware of block size, all allocations were concealed and administered in correct order with no deviations. Following recruitment, a different team member (SSP) opened the envelope to reveal the BP intervention level. 


\section{Intervention}

Infants were randomised into one of three arms. They received BP support if they met these criteria:

i. $\quad$ Active arm: Mean arterial BP (MABP) was supported if it fell below 30 mmHg for more than 15 consecutive minutes

ii. Moderate arm: MABP was supported if it fell below the infants gestational age in mmHg for more than 15 consecutive minutes

iii. Permissive arm: Cardiovascular support was given for clinical evidence of impaired tissue perfusion assessed by the clinician (poor skin perfusion with capillary refill time $>3$ seconds, urine output $<1 \mathrm{ml} / \mathrm{kg} /$ hour from weighing of urine, worsening base excess $(<-8 \mathrm{mmol} / \mathrm{L})$ or rising lactate $(>2 \mathrm{mmol} / \mathrm{L})$ on blood gas analysis) and/or if MABP fell below a lower safety net value of 19 mmHg for more than 15 consecutive minutes. Infants also qualified for cardiovascular support if MABP remained $\geq 19 \mathrm{mmHg}$ with evidence of impaired tissue perfusion.

The threshold of $19 \mathrm{mmHg}$ in the Permissive arm was arbitrarily chosen, as the lowest value which clinicians accepted, giving separation from the lowest Moderate arm value.

Infants received the same BP management across the three arms of the study. The therapeutic goal of cardiovascular support was to achieve a MABP (or measure of tissue perfusion) which was greater than the threshold at which the infant became eligible for the intervention. 


\section{Circulatory management}

BP management prior to randomisation followed the unit's established guidelines, the same as in the Active arm. Perinatal management included antenatal steroids, a modest delay in umbilical cord clamping (30-60sec), with no routine intravascular volume before NICU.

A written protocol tailored for each gestation and study arm was at the cotside to guide staff, with BP intervention levels. Apart from this target, the guideline (Appendix A) was similar to standard NICU policy. Key elements included triggers for evaluation (BP, tachycardia, impaired perfusion), basic stabilisation, and a maximum of 10-20mls/kg intravascular volume. First-line inotropes were dopamine for low BP, and dobutamine for poor perfusion. If inotropes were escalated, clinical re-evaluation was performed, usually involving functional echocardiography to target treatment (options included intravascular volume, second inotropes, pressors, hydrocortisone, pulmonary vasodilators or PDA treatment). Clinician discretion in starting inotropes was allowed, assessing overall clinical status, so starting therapy before breaching BP threshold was not a protocol deviation. Clinicians were not blinded to the various intervention groups.

All infants had amplitude integrated electroencephalography (aEEG), right common carotid artery blood flow (RCCAF) volume, left ventricular output (LVO) and superior mesenteric artery (SMA) velocity measurements on days 1 and 3.

\section{Blood pressure monitoring}

Infants with both invasive and non-invasive BP monitoring were included to reflect clinical practice, where invasive BP monitoring is not universally instituted. The decision to monitor BP invasively was made by the responsible clinician. 
Invasive BP measurements were obtained from transducers attached to umbilical arterial catheters in a high position (thoracic vertebral body level T6-10) calibrated daily at the mid-axillary line. Precautions were taken to ensure good quality waveforms, displayed using GE Healthcare systems (Carescape Monitor B850) and downloaded onto a laptop every 10 seconds for the first week.

Non-invasive BP was performed every 15 minutes if below threshold, otherwise hourly for the first 12 hours and 4-hourly thereafter, using the GE oscillometric method with appropriate sized cuffs.

\section{Physiological measurements}

Blood flow measurements were performed using Doppler ultrasound and diameter measurement with a Phillips iE33 system (Bothwell, USA). A 4-12 MHz transducer was used for LVO using an apical view ${ }^{20}$ which correlates well with phase contrast MRI. ${ }^{21}$ A 7-15 MHz probe was used to measure RCCAF volume using an established method $^{22}$ shown to have good reproducibility. A 5-8 MHz transducer was used for SMA velocity. ${ }^{23}$

\section{Electroencephalography recording}

aEEG was recorded for 72 hours using a 2-channel BRM3 monitor (BrainZ Instruments, Canada). Hydrogel electrodes were placed in the centro-parietal regions (C3-P3, C4-P4) bilaterally according to the 10-20 system ${ }^{24}$. A 2-hour artefact and seizure free electroencephalogram trace, recorded before and after RCCAF measurement was analysed. Cross-cerebral aEEG was assessed for median, minimum and maximum amplitude. The discontinuity of the trace was calculated from the raw 
electroencephalogram (threshold 20 microvolts) using software ${ }^{25}$ in MATLAB ${ }^{\circledR}$ (The MathWorks Inc., USA).

\section{Classification of cerebral pathology on Cranial ultrasound scan (CrUSS)}

Unit policy was for CrUSS on Days 1,3,7,14,28 and at 36 weeks corrected age, or before discharge. All first week CrUSS, and the CrUSS closest to 36 weeks, were reviewed independently by two neonatal consultants (STK \& AKS) blind to allocation. Periventricular haemorrhage (PVH) was classified on the Papile scale ${ }^{26}$; later findings included porencephalic cyst, periventricular leukomalacia and ventricular dilatation.

\section{Clinical and Physiological Outcomes}

Predefined primary outcomes were MABP during the first week, and the use/duration of inotropes. Predefined secondary clinical outcomes included death or parenchymal brain abnormality on cerebral ultrasound, death before discharge home from hospital, periventricular leukomalacia, parenchymal or other periventricular haemorrhage, necrotising enterocolitis using Bell’s staging criteria and localised intestinal perforation, treatment for patent ductus arteriosus, maximum serum creatinine and potassium in the first 2 weeks, duration of respiratory support, oxygen dependency or respiratory support at 36 weeks post-conceptual age, postnatal steroids including hydrocortisone.

Physiological outcomes were RCCAF, SMA blood flow velocity, LVO and EEG measurements, as detailed above.

\section{Statistical analysis}

Sample size calculations were performed for $80 \%$ power at a $5 \%$ significance level, with 2-sided tests in a 3-armed study design, without correction for multiple 
comparisons. For the primary outcome of inotrope usage, previous data showed rates of $15 \%$ where permissive hypotension was practiced ${ }^{27}$ and $68 \%$ in our unit using active management (unpublished data). Sample size was calculated from a 15\% vs 65\% difference in proportions, with continuity correction, requiring 18 patients in each group (STATA, version 14, StataCorp 2015). Inotrope usage was used in these calculations as data was not available for duration of inotrope usage nor MABP during the first week. A planned study size of 20 patients in each study arm was used to allow for attrition or uneven recruitment.

To reduce multiple pairwise testing, data were examined for effects across three treatment arms, using tests for ordered effects (assuming Active $>$ Moderate $>$ Permissive). For normally distributed continuous variables this used ANOVA and predefined contrasts, for non-normal data the Jonckheere-Terpstra test. For categorical outcomes Chi-squared test with Linear-by-Linear association was used, or Fisher's exact test for predicted cell numbers $<5$.

After excluding artefacts, invasive BP during the first 3 days was analysed in a mixed effects general linear model, with random effects included in the intercept of the model to account for between-baby variation, and gestation modelled as a fixed effect by completed week of gestation, and time as a fixed effect by 4-hourly epochs.

As an exploratory pilot trial, tables give statistical significance without correction for multiple testing, but also indicate whether $p$-values remain significant using the Benjamini-Hochberg correction ${ }^{28}$, with a false discovery rate of $10 \%$ for each analysis (clinical outcomes and physiological outcomes).

This study received approval from the London-Surrey Borders Research Ethics 
Committee (reference 12/LO/1553), NHS National Research Ethics Service. 


\section{RESULTS}

\section{Patient characteristics}

The trial ended after planned recruitment of 60 infants between February 2013 and April 2015. This represented 45\% of 134 NICU admissions within eligible gestation and postnatal age ranges (Figure 1). In two patients clinicians requested that the patient should not be recruited because of a clinical and echocardiographic diagnosis of persistent pulmonary hypertension of the newborn (PPHN). There were no differences between those recruited, and those who were not, in mean gestation (25.8 vs 26.0 weeks), inborn proportion (70\% vs $64 \%$ ) or mortality (15\% vs $19 \%$ ).

No parents gave antenatal assent. All those recruited were followed up and included in the analysis with no protocol deviations. Clinician discretion in starting inotropes was allowed, assessing overall clinical status, so starting therapy before breaching BP threshold was not a protocol deviation. Median randomisation age was 8 hours (range 1-12). Randomised groups were comparable (Table 1).

\begin{tabular}{|c|c|c|c|c|}
\hline Patient characteristics & $\begin{array}{l}\text { Active arm } \\
\quad(n=19)\end{array}$ & $\begin{array}{l}\text { Moderate arm } \\
\quad(n=20)\end{array}$ & $\begin{array}{l}\text { Permissive arm } \\
\quad(n=21)\end{array}$ & $\begin{array}{c}p \text { - } \\
\text { value }\end{array}$ \\
\hline Gestational age in weeks & $25.7(23.4-28.9)$ & $25.8(23.3-28.7)$ & $25.6(23.7-28.7)$ & .91 \\
\hline Birth weight in grams & $760(580-1000)$ & $810(470-1180)$ & $790(540-1470)$ & .42 \\
\hline Females, $n(\%)$ & $11(58 \%)$ & $8(40 \%)$ & $11(52 \%)$ & .75 \\
\hline $\begin{array}{l}\text { Antenatal steroids, } n(\%) \\
\text { No steroids } \\
\text { Two doses of steroids }\end{array}$ & $\begin{array}{c}1(5 \%) \\
16(84 \%)\end{array}$ & $\begin{array}{c}2(10 \%) \\
14(70 \%)\end{array}$ & $\begin{array}{c}1(5 \%) \\
15(71 \%)\end{array}$ & .84 \\
\hline $\begin{array}{l}\text { Mothers who received anti- } \\
\text { hypertensive medication, } n \\
\text { (\%) }\end{array}$ & $4(21 \%)$ & $4(20 \%)$ & $2(10 \%)$ & .57 \\
\hline $\begin{array}{l}\text { Mothers who received } \\
\text { magnesium sulphate, } n(\%) \\
\text { No data available, } n(\%)\end{array}$ & $\begin{array}{l}5(26 \%) \\
14(74 \%) \\
\end{array}$ & $\begin{array}{l}5(25 \%) \\
15(75 \%) \\
\end{array}$ & $\begin{array}{l}1(5 \%) \\
20(95 \%) \\
\end{array}$ & .58 \\
\hline $\begin{array}{l}\text { Mode of delivery, } n(\%) \\
\text { Vaginal delivery } \\
\text { Caesarean section }\end{array}$ & $\begin{array}{c}15(79 \%) \\
4(21 \%)\end{array}$ & $\begin{array}{c}17(85 \%) \\
3(15 \%)\end{array}$ & $\begin{array}{c}20(95 \%) \\
1(5 \%)\end{array}$ & .28 \\
\hline $\begin{array}{l}\text { Apgar score } \\
1 \mathrm{~min} \\
5 \mathrm{~min}\end{array}$ & $\begin{array}{c}5(0-9) \\
8(1-10)\end{array}$ & $\begin{array}{c}5(1-9) \\
7(4-10)\end{array}$ & $\begin{array}{c}3(1-9) \\
7(3-10)\end{array}$ & $\begin{array}{l}.21 \\
.96\end{array}$ \\
\hline
\end{tabular}




\begin{tabular}{|l|c|c|c|c|}
\hline $\begin{array}{l}\text { Temperature on admission } \\
\text { to NICU }\end{array}$ & $36.9(33.4-37.8)$ & $36.9(35.5-37.5)$ & $36.8(35.8-38.1)$ & .65 \\
\hline CRIB II scores & $13(8-17)$ & $12(8-16)$ & $12(5-15)$ & .10 \\
\hline $\begin{array}{l}\text { Invasive ventilation, } n(\%) \\
\text { Day 1 }\end{array}$ & $18(95 \%)$ & $18(90 \%)$ & $20(95 \%)$ & .84 \\
Day 3 & $16(84 \%)$ & $15(75 \%)$ & $11(52 \%)$ & .08 \\
\hline Mean airway pressure, cms & & & & \\
of $\mathrm{H}_{2} \mathrm{O}$ & $8(4-11)$ & $9(5-11)$ & $7(5-9)$ & .05 \\
Day 1 & $8(5-15)$ & $8(6-15)$ & $7(4-10)$ & .02 \\
Day 3 & & & & \\
\hline
\end{tabular}

Table 1: Perinatal characteristics at the time of recruitment and randomisation. Where not specified all figures are median (range). None of the differences were significant after correction for multiple testing.

\section{Blood Pressure and Cardiovascular Support}

Initial analysis of intermittent, staff-recorded BP, including both invasive and noninvasive measurements, did not show MABP differences between groups. A MABP $<19 \mathrm{mmHg}$ was found in 4 infants in the Permissive, none in the Moderate and one in the Active arm. Non-invasive MABP measurement was on average 11mmHg higher than the preceding invasive value; the $95 \%$ limits of agreement between staff-recorded and continuously downloaded invasive BP was $+/-6 \mathrm{mmHg}$, with no systematic bias. Detailed analysis of BP was therefore restricted to 51 infants (85\%) with continuous invasive BP monitoring between 12-72 hours.

On the first postnatal day, invasive BP was highest in the Active arm, and was most stable in this group, with no significant effects in any time epoch during the first 3 days (Figure 2). In the Moderate arm, BP was significantly reduced at 12-15 hours of age (-3.1mmHg, 95\%CI -5.2 to -1.0$)$, and overshot to become significantly elevated at 32-39 hours of age (+3.5mmHg at 36-39 hrs, 95\%CI 1.3 to 5.6). In the Permissive arm, BP was significantly reduced at 8-19 hours of age $(-5.2 \mathrm{mmHg}$ at $12-15 \mathrm{hrs}$, 95\%CI -6.3 to -4.1$)$, with a short period of elevation at 24-27 hours $(+1.7 \mathrm{mmHg}$, 95\%CI 0.6 to 2.7). After 72 hours, there were no major differences in BP between the groups (Supplementary table 1). 
Inotropic support was given most frequently, and for longest duration, in the Active arm (79\% inotropes, mainly dopamine), and was used least in the Permissive arm (48\%, $p=0.05$ ). Although 12/35 (34\%) of the patients receiving inotropes had their treatment started before randomisation, this did not differ between groups, and after randomisation inotropes were started significantly more often in the Active arm. Intravascular volume use prior to inotropes was similar in different study arms (Active 7/15, Moderate 8/10, Permissive 8/10). In the Permissive arm, 3 infants had a MABP $<19 \mathrm{mmHg}$ recorded in the hour that inotropes were started. There were no differences in hydrocortisone use, intravascular volume expansion or in PDA treatment (Table 2).

\begin{tabular}{|c|c|c|c|c|c|}
\hline & $\begin{array}{l}\text { Active arm } \\
n=19\end{array}$ & $\begin{array}{l}\text { Moderate arm } \\
n=20\end{array}$ & $\begin{array}{c}\text { Permissive arm } \\
n=21\end{array}$ & $p$-value & $\mathbf{P}(\mathrm{c})$ \\
\hline \multicolumn{6}{|c|}{ Clinical cardiovascular treatment: Intravascular volume expansion } \\
\hline Pre-randomisation, (ml/kg) & $0(0-10)$ & $0(0-10)$ & $0(0-8)$ & $.94^{\mathrm{J}}$ & \\
\hline $\begin{array}{l}\text { During } 72 \text { hours post } \\
\text { randomisation, (ml/kg) }\end{array}$ & $30(15-50)$ & $15(0-33)$ & $23(0-49)$ & $.30^{\mathrm{J}}$ & \\
\hline $\begin{array}{l}\text { Total volume expansion given in } \\
\text { the first week }(\mathrm{ml} / \mathrm{kg})\end{array}$ & $65(15-85)$ & $40(0-68)$ & $35(0-89)$ & $.28^{\mathrm{J}}$ & \\
\hline \multicolumn{6}{|c|}{ Clinical cardiovascular treatment: Inotropic agents } \\
\hline Inotropic support $(n)$ & $15(79 \%)$ & $10(50 \%)$ & $10(48 \%)$ & .05 & \\
\hline $\begin{array}{l}\text { Inotrope usage by gestation }(n) \\
23-24 \text { weeks } \\
25-26 \text { weeks } \\
27-28 \text { weeks }\end{array}$ & $\begin{array}{r}5 / 5(100 \%) \\
8 / 10(80 \%) \\
2 / 4(50 \%)\end{array}$ & $\begin{array}{c}5 / 6(83 \%) \\
4 / 10(40 \%) \\
1 / 4(25 \%)\end{array}$ & $\begin{array}{r}5 / 5(100 \%) \\
4 / 11(36 \%) \\
1 / 5(20 \%)\end{array}$ & - & \\
\hline $\begin{array}{l}\text { Age inotropes started (hours) } \\
\text { Started before randomised }(n) \\
\text { Started after randomised }(n)\end{array}$ & $\begin{array}{c}8(5-12) \\
2(11 \%) \\
13(68 \%) \\
\end{array}$ & $\begin{array}{c}5(4-13) \\
6(30 \%) \\
4(20 \%) \\
\end{array}$ & $\begin{array}{c}8(4-92) \\
4(19 \%) \\
6(29 \%) \\
\end{array}$ & $\begin{array}{l}.82^{\mathrm{J}} \\
.53 \\
.01 \\
\end{array}$ & * \\
\hline $\begin{array}{l}\text { Duration of inotropes (hours) } \\
\text { All infants } \\
\text { Infants receiving inotropes }\end{array}$ & $\begin{array}{l}44(20-153) \\
56(31-197)\end{array}$ & $\begin{array}{c}7(0-73) \\
68(37-118)\end{array}$ & $\begin{array}{c}0(0-39) \\
39(34-42)\end{array}$ & $\begin{array}{l}.01^{\mathrm{J}} \\
.07^{\mathrm{J}}\end{array}$ & * \\
\hline $\begin{array}{l}\text { Inotropic agents used }(n) \\
\text { Dopamine } \\
\text { Dobutamine }\end{array}$ & $\begin{array}{r}15(79 \%) \\
2(10 \%)\end{array}$ & $\begin{array}{c}10(50 \%) \\
1(5 \%)\end{array}$ & $\begin{array}{l}8(43 \%) \\
3(14 \%)\end{array}$ & $\begin{array}{l}.03 \\
.77^{\mathrm{F}}\end{array}$ & \\
\hline
\end{tabular}




\begin{tabular}{|c|c|c|c|c|}
\hline $\begin{array}{l}\text { Maximum dose of dopamine - } \\
\mathrm{mcg} / \mathrm{kg} / \mathrm{min}\end{array}$ & $17(10-20)$ & $12(10-16)$ & $11(10-14)$ & $.09^{\mathrm{A}}$ \\
\hline \multicolumn{5}{|c|}{ Clinical cardiovascular treatment: other cardioactive drugs and monitoring } \\
\hline Hydrocortisone for hypotension $(n)$ & $4(21 \%)$ & $3(15 \%)$ & $4(19 \%)$ & $.92^{\mathrm{F}}$ \\
\hline $\begin{array}{l}\text { Patent ductus arteriosus }(n) \\
\text { Medical treatment (COX } \\
\text { inhibitor) } \\
\text { Surgical ligation }\end{array}$ & $\begin{array}{l}7(37 \%) \\
2(10 \%)\end{array}$ & $\begin{array}{l}9(45 \%) \\
2(10 \%)\end{array}$ & $\begin{array}{l}8(38 \%) \\
5(24 \%)\end{array}$ & $\begin{array}{l}.95 \\
.48^{\mathrm{F}}\end{array}$ \\
\hline Invasive BP monitoring & $17(89 \%)$ & $18(90 \%)$ & $16(76 \%)$ & $.48^{\mathrm{F}}$ \\
\hline $\begin{array}{l}\text { Duration of umbilical arterial } \\
\text { catheterisation - hours }\end{array}$ & $\begin{array}{c}105 \\
(68-232)\end{array}$ & $\begin{array}{c}108 \\
(55-227)\end{array}$ & $\begin{array}{c}79 \\
(45-187)\end{array}$ & $.22^{\mathrm{J}}$ \\
\hline
\end{tabular}

\section{Physiological characteristics on day 1 and 3 of life: Circulatory variables}

Cardiac output (left ventricular output, $\mathrm{ml} / \mathrm{kg} / \mathrm{min}$ )

\begin{tabular}{l|l|l|l|l|} 
Day 1 & $166(159-220)$ & $160(125-195)$ & $181(144-202)$ & $.58^{\mathrm{A}}$ \\
Day 3 & $210(147-256)$ & $220(164-250)$ & $200(172-251)$ & $.67^{\mathrm{A}}$ \\
\hline
\end{tabular}

Mean arterial (invasive and non-invasive) blood pressure (mmHg) during echocardiography

\begin{tabular}{l|l|l|l|l|} 
Day 1 & $36(33-38)$ & $32(29-35)$ & $34(28-37)$ & $.65^{\mathrm{A}}$ \\
Day 3 & $36(33-39)$ & $33(29-41)$ & $40(32-51)$ & $.33^{\mathrm{A}}$ \\
\hline
\end{tabular}

Mean arterial (invasive) blood pressure (mmHg) during echocardiography

\begin{tabular}{|l|l|l|l|l|} 
Day 1 & $35(32-37)$ & $32(29-35)$ & $31(27-35)$ & $.01^{\mathrm{A}}$ \\
Day 3 & $34(32-36)$ & $32(28-35)$ & $33(30-39)$ & $.56^{\mathrm{A}}$ \\
\hline
\end{tabular}

Common carotid artery blood flow ( $\mathrm{ml} / \mathrm{kg} / \mathrm{min})$

\begin{tabular}{|l|c|c|c|c|} 
Day 1 & $12(10-14)$ & $12(8-14)$ & $12(9-16)$ & $.41^{\mathrm{A}}$ \\
Day 3 & $15(12-18)$ & $13(12-15)$ & $15(11-20)$ & $.66^{\mathrm{A}}$ \\
\hline
\end{tabular}

Superior mesenteric artery blood flow velocity (mean of peak velocity envelope - $\mathrm{cm} / \mathrm{s}$ )

\begin{tabular}{|l|l|l|l|l|} 
Day 1 & $21(16-29)$ & $29(15-35)$ & $21(17-28)$ & $.84^{\mathrm{A}}$ \\
Day 3 & $21(14-27)$ & $30(24-40)$ & $26(22-31)$ & $.04^{\mathrm{A}}$ \\
\hline
\end{tabular}

\section{Patent ductus arteriosus}

Patent ductus arteriosus (PDA) present $(n(\%))$

\begin{tabular}{|l|l|l|l|l|} 
Day 1 & $18(95 \%)$ & $19(95 \%)$ & $17(81 \%)$ & $.35^{\mathrm{F}}$ \\
Day 3 & $12(63 \%)$ & $13(68 \%)$ & $15(71 \%)$ & .58 \\
\hline
\end{tabular}

PDA maximum (colour) diameter (mm)

\begin{tabular}{|l|c|c|c|c|} 
Day 1 & $1.6(1.2-1.9)$ & $1.3(1-1.8)$ & $1.3(1.2-1.8)$ & $.13^{\mathrm{A}}$ \\
Day 3 & $1.1(0-1.6)$ & $1.1(0-1.6)$ & $1.2(0-1.5)$ & $.94^{\mathrm{A}}$ \\
\hline
\end{tabular}

PDA maximum velocity $(\mathrm{m} / \mathrm{sec})$

\begin{tabular}{|l|c|c|c|c|c|}
\hline Day 1 & $1.4(0.8-1.6)$ & $1.3(0.9-1.6)$ & $1.2(0.4-1.9)$ & $.92^{\mathrm{A}}$ & \\
Day 3 & $0.6(0-1.7)$ & $0.9(0-1.4)$ & $0.7(0-1.5)$ & $.70^{\mathrm{A}}$ & \\
\hline
\end{tabular}

PDA flow pattern $(n(\%))$ as per Su et $\mathrm{al}^{29}$

\begin{tabular}{|c|l|l|l|l|l|}
\hline Day 1: Growing pattern & $6(50 \%)$ & $3(25 \%)$ & $3(25 \%)$ & $.39^{\mathrm{F}}$ & \\
Pulsatile pattern & $9(39 \%)$ & $9(39 \%)$ & $5(22 \%)$ & & \\
\hline Day 3: Growing pattern & $2(40 \%)$ & $2(40 \%)$ & $1(20 \%)$ & $.80^{\mathrm{F}}$ & \\
\hline
\end{tabular}




\begin{tabular}{|c|c|c|c|c|}
\hline Pulsatile pattern & $7(32 \%)$ & $7(32 \%)$ & $8(36 \%)$ & \\
\hline \multicolumn{5}{|c|}{ Physiological characteristics on day 1 and 3 of life: Electroencephalographic variables } \\
\hline \multicolumn{5}{|c|}{ Maximum aEEG amplitude in $\mu \mathrm{V}$} \\
\hline Day 1 & $12.0(9.8-14.3)$ & $10.0(7.3-14.4)$ & $13.1(9.6-15.5)$ & $.45^{\mathrm{A}}$ \\
\hline Day 3 & $15.0(8.3-20.6)$ & $16.0(10.0-19.8)$ & $13.0(12.9-18.8)$ & $.82^{\mathrm{A}}$ \\
\hline \multicolumn{5}{|c|}{ Minimum aEEG amplitude in $\mu \mathrm{V}$} \\
\hline Day 1 & $3.1(2.8-3.9)$ & $2.5(2.0-3.7)$ & $3.5(2.5-3.9)$ & $.63^{\mathrm{A}}$ \\
\hline Day 3 & $3.5(2.4-5.1)$ & $4.0(2.9-5.2)$ & $4.2(3.4-5.3)$ & $.87^{\mathrm{A}}$ \\
\hline \multicolumn{5}{|c|}{ Median discontinuity in seconds per one minute epoch } \\
\hline Day 1 & $20(15-26)$ & $25(17-37)$ & $17(9-30)$ & $.41^{\mathrm{A}}$ \\
\hline Day 3 & $9.5(1.5-31.9)$ & $11(6-23.7)$ & $8.5(0-18.6)$ & $.49^{\mathrm{A}}$ \\
\hline
\end{tabular}

Table 2: Cardiovascular treatments, circulatory measurements and electroencephalographic variables. Values are number (\%) or median (IQR), with statistical tests performed for ordered levels (Chi-squared test with linear-by-linear association and Jonckheere-Terpstra test (J)), ANOVA with contrasts (A) or Fisher's exact test (F)). Starting inotropic therapy after randomization, and median duration of inotropic therapy for all patients, were the only variables that remained significant after Benjamini-Hochberg correction for multiple testing (indicated by * under $\mathrm{P}(\mathrm{c})$ ).

\section{Hemodynamic and electroencephalographic variables}

Detailed echocardiography and aEEG were performed simultaneously on Days 1 and 3 (median 18 and 77 hours). Cardiac output, ductal shunt, RCCAF, aEEG variables did not differ significantly between study arms (Table 2).

\section{Clinical outcomes}

There were no significant differences in clinical outcomes, including mortality, duration of care, respiratory or gastrointestinal complications, retinopathy or renal variables (Supplementary table 2).

\section{Cranial ultrasound findings}

The review of CrUSS findings reclassified clinical team reports in 17\% cases. There was blinded agreement in 92\%, and full agreement with minor adjustments after discussion. 
Parenchymal PVH occurred in only 2/60 infants. A normal CrUSS was found most often in the Active arm, and combined rates of Grade 2-4 PVH were highest in the Moderate arm (Active 0\%, Moderate 30\%, Permissive 5\%; Fisher's exact $p=0.008$ ). The predefined outcome of death or parenchymal brain abnormality did not differ between groups (Table 3).

\begin{tabular}{|c|c|c|c|c|}
\hline & $\begin{array}{l}\text { Active } \\
n=19\end{array}$ & $\begin{array}{c}\text { Moderate } \\
n=20\end{array}$ & $\begin{array}{c}\text { Permissive } \\
n=21\end{array}$ & $p$-value \\
\hline Normal CrUSS on Day 1 & $16 / 18(89 \%)$ & $14 / 19(74 \%)$ & $16 / 18(89 \%)$ & .47 \\
\hline \multicolumn{5}{|c|}{ Periventricular Haemorrhage in first week of life } \\
\hline Normal - No PVH & $16(84 \%)$ & $12(60 \%)$ & $16(76 \%)$ & .22 \\
\hline $\begin{array}{l}\text { Subependymal } \\
\text { Haemorrhage }\end{array}$ & $3(16 \%)$ & $2(10 \%)$ & $4(19 \%)$ & - \\
\hline $\begin{array}{l}\text { Intraventricular } \\
\text { haemorrhage (no } \\
\text { dilatation) }\end{array}$ & 0 & $3(15 \%)$ & $1(5 \%)$ & - \\
\hline $\begin{array}{l}\text { Intraventricular } \\
\text { haemorrhage with } \\
\text { dilatation }\end{array}$ & 0 & $1(5 \%)$ & 0 & - \\
\hline $\begin{array}{l}\text { Haemorrhagic } \\
\text { parenchymal infarct }\end{array}$ & 0 & $2(10 \%)$ & 0 & - \\
\hline \multicolumn{5}{|l|}{ Late findings } \\
\hline Normal & $15(94 \%)$ & $15(83 \%)$ & $14(88 \%)$ & .86 \\
\hline $\begin{array}{l}\text { Cystic Periventricular } \\
\text { Leukomalacia }\end{array}$ & 0 & 0 & 0 & - \\
\hline Porencephalic cyst & 0 & 2 & 1 & - \\
\hline Ventricular dilatation & 1 & 1 & 1 & - \\
\hline \multicolumn{5}{|c|}{ Combined early and late findings } \\
\hline $\begin{array}{l}\text { Death or parenchymal } \\
\text { brain abnormality }\end{array}$ & $4(21 \%)$ & $4(20 \%)$ & $3(14 \%)$ & .84 \\
\hline $\begin{array}{l}\text { Any abnormality on early } \\
\text { or late scans }\end{array}$ & $4(21 \%)$ & $8(40 \%)$ & $6(29 \%)$ & .44 \\
\hline
\end{tabular}

Table 3: Cranial ultrasound findings, assessed blinded to study allocation. There were no differences in pre-specified outcomes (Fisher's exact test; all infants with cranial 
ultrasound abnormalities had invasive BP monitoring on Day 1 and only two infants had non-invasive BP monitoring on Day 3. 


\section{DISCUSSION}

This is the first prospective randomised trial of BP intervention levels in preterm neonates to report its findings, demonstrating feasibility of such a study. This design achieved separation between study arms in inotropic therapy and BP levels. However, differences were less than might have been supposed, with major differences in invasively measured BP found mainly in the first 2 days. Differences in inotrope usage mainly occurred at 25-26 weeks gestation; most infants below this received inotropes, and most infants at higher gestations did not. The differences between intermittent staff BP recordings and continuous invasive measurements, suggest that future studies should concentrate on infants with invasive monitoring, using automatically downloaded data.

Differences in BP between groups were not reflected in cardiac output or EEG variables measured at fixed time points, but these measurements may have missed the period when BP differed most. This pilot study was not powered to detect differences in major clinical outcomes, and reassuringly there were no effects on mortality.

There were no statistically significant differences in pre-specified cranial ultrasound findings between study arms. However, it was notable that very few infants in the Active arm had significant intracranial pathology, only three had subependymal PVH, and none had parenchymal lesions. The highest rates of periventricular hemorrhage were found in the Moderate arm. Fluctuating pressure passive cerebral blood flow in sick preterm infants is associated with $\mathrm{PVH}^{3031}$, so it is biologically plausible that the instability of BP in the Moderate arm, could provide a mechanism for more intracranial pathology in this group.

The design of randomising patients to different BP intervention levels, but instituting 
the same management in all study arms, encouraged parents and clinicians to let infants participate in the study, with reasonable recruitment. Added safety by measuring cardiac output may have reassured clinicians. This contrasts with difficult recruitment in a trial using fixed intervention levels, with patients randomised to treatment or placebo. ${ }^{32}$ We did not prevent clinical staff from starting inotropes before recruitment or when they considered it clinically indicated, even if the BP criteria had not been breached for 15 minutes. As a significant proportion of infants had inotropes started before randomisation, we probably underestimated the differences in inotrope use which these policies would have produced if instituted from birth, even if the policy subsequently modified inotrope duration and dose. The reasons for starting treatment appear to have been based on a number of factors including BP and perfusion markers. Future studies could explore these reasons further, and may find larger differences using cluster randomised trials, or with a waiver of consent, if considered acceptable to parents.

As an exploratory pilot, this study had limited power for clinical outcome effects. By analysing cardiovascular and EEG measurements at fixed time-points, we may have missed perfusion disturbances occurring at different times in each patient. Future studies of physiological variables should expect the greatest differences at 12-36 hours of age. Infants did not have pre-trial entry cranial/cardiac ultrasound scans but the numbers of infants with normal CrUSS on day 1 did not differ significantly between groups. Given the benefits of delayed cord clamping, ${ }^{33} 34$ a modest delay in cord clamping (30-60 seconds) was the standard of care but the exact time of delay was not recorded. Inotropic support pre-randomisation was relatively higher in the Moderate arm but not statistically different between the arms. Though the effect of delayed cord clamping on inotropic support is well described, this does not account for the trend in inotropic support post-randomisation, where infants in the Active arm received the 
most inotropic support. Volume expansion was given prior to inotropic support in the majority of infants, 23 out of 35 (66\%), did not differ between study arms, and was not related to delayed cord clamping. This study only examined a single aspect of cardiovascular management, BP intervention level. There are many other issues of equal importance, to be studied independently, including detection of altered perfusion, optimum therapies for hypotension or impaired perfusion, treatment of the ductus arteriosus, and whether treatment should be individualised.

We recommend that future studies should concentrate on infants with invasive BP monitoring, with data preferably downloaded for analysis. RCTs may be clinically acceptable if there is a safety-net, using echocardiography or other non-invasive methods, to detect severe cardiovascular compromise. Important outcomes can include death or neurodevelopmental impairment, but should also examine periventricular haemorrhage, using blinded classification of CrUSS. It may be worth examining approaches which are either more or less active than those in common use. 


\section{Declaration of interest}

David Wertheim is an inventor on a patent US5181520 “Method and apparatus for analysing an electro-encephalogram”. The other authors have no conflicts of interest to disclose.

\section{Acknowledgements}

We would like to thank Dr. N. Aladangady and Dr. A. Groves for their input into the study data monitoring committee. We thank the parents and infants who participated in this study and also all the doctors and nursing staff of the neonatal intensive care unit at the Royal London Hospital. We are grateful to the parents who raised funds to support this study, and to Barts Charity who administered this funding.

\section{What is already known on this topic:}

- The criterion for supporting low BP in extremely preterm infants remains controversial.

- A gestation based approach to supporting BP remains the most common method.

- The relation between low BP and adverse outcomes, and the safety of anti-hypotensive treatment remains unknown.

\section{What this study adds:}

- It is feasible to carry out a 3-arm RCT investigating BP intervention levels in extremely preterm infants.

- The majority of infants below 25 weeks gestation received inotropes irrespective of the arm they were randomised to.

- The BP threshold used to trigger treatment affects achieved BP and inotrope usage. 


\section{REFERENCES}

1. Batton BL, L.; Newman, N.S.; Das, A.; Watterberg, K.L.; Yoder, B.A.; Faix, R.G.; Laughon, M.M.; Stoll, B.J.; Van Meurs, K.P.; et al. Use of antihypotensive therapies in extremely preterm infants. Pediatrics 2013, 131, e1865-e1873. Pediatrics 2013 2013;131:e1865-e73.

2. Laughon M, Bose C, Allred E, et al. Factors Associated With Treatment for Hypotension in Extremely Low Gestational Age Newborns During the First Postnatal Week. Pediatrics 2007;119(2):273-80. doi: 10.1542/peds.2006-1138

3. Sehgal A, Osborn D, McNamara PJ. Cardiovascular support in preterm infants: a survey of practices in Australia and New Zealand. Journal of paediatrics and child health 2012;48(4):317-23. doi: 10.1111/j.1440-1754.2011.02246.x [published Online First: 2011/11/17]

4. Bhojani S. BJ, Rahman M.M. Management of neonatal hypotension - a national questionnaire survey. . Infant 2010;6(5):152-54.

5. Stranak Z, Semberova J, Barrington K, et al. International survey on diagnosis and management of hypotension in extremely preterm babies. Eur J Pediatr 2014;173(6):793-8. doi: 10.1007/s00431-013-2251-9

6. Al-Aweel IP, D.M.; Rubin, L.P.; Shah, B.; Weisberger, S.; Richardson, D.K. Variations in prevalence of hypotension, hypertension, and vasopressor use in NICUs. Journal of Perinatology 2001;21(5):272-78.

7. Faust K, Hartel C, Preuss M, et al. Short-term outcome of very-low-birthweight infants with arterial hypotension in the first $24 \mathrm{~h}$ of life. Arch Dis Child Fetal Neonatal Ed 2015 doi: 10.1136/archdischild-2014-306483

8. Alderliesten T, Lemmers PM, van Haastert IC, et al. Hypotension in preterm neonates: low blood pressure alone does not affect neurodevelopmental outcome. The Journal of pediatrics 2014;164(5):986-91. doi: 10.1016/j.jpeds.2013.12.042

9. Logan JW, O'Shea TM, Allred EN, et al. Early postnatal hypotension and developmental delay at 24 months of age among extremely low gestational age newborns. Archives of disease in childhood Fetal and neonatal edition 2011;96(5):F321-8. doi: 10.1136/adc.2010.183335 
10. Short BL, Van Meurs K, Evans JR, et al. Summary proceedings from the cardiology group on cardiovascular instability in preterm infants. Pediatrics 2006;117(3 Pt 2):S34-9. doi: 10.1542/peds.2005-0620F

11. Lopez SL, Leighton, J.O., Walther, F.J. Supranormal Cardiac Output in the Dopamine and Dobutamine Dependent Preterm Infant. .ös:Pediatr Cardiology 1997;18:292-96.

12. Pladys P, Wodey E, Beuchee A, et al. Left ventricle output and mean arterial blood pressure in preterm infants during the 1st day of life. European journal of pediatrics 1999;158(10):817-24. [published Online First: 1999/09/15]

13. Kluckow M, Evans N. Relationship between blood pressure and cardiac output in preterm infants requiring mechanical ventilation. J Pediatr 1996;129(4):506-12. [published Online First: 1996/10/01]

14. Seri I, Evans J. Controversies in the diagnosis and management of hypotension in the newborn infant. Current Opinion in Pediatrics 2001;13(2):116-23.

15. group Jw. Development of audit measures and guidelines for good practice in the management of neonatal respiratory distress syndrome. Report of a Joint Working Group of the British Association of Perinatal Medicine and the Research Unit of the Royal College of Physicians. Archives of disease in childhood 1992;67(10 Spec No):1221-7.

16. Dempsey EM, Barrington KJ. Diagnostic criteria and therapeutic interventions for the hypotensive very low birth weight infant. Journal of perinatology 2006;26(11):677-81. doi: 10.1038/sj.jp.7211579

17. Miall-Allen VM, De Vries, L. S.; Whitelaw, A.G.L. Mean arterial blood pressure and neonatal cerebral lesions. Archives of disease in childhood 1987;62(10):1068-69.

18. Munro MJ, Walker AM, Barfield CP. Hypotensive extremely low birth weight infants have reduced cerebral blood flow. Pediatrics 2004;114(6):1591-6. doi: 10.1542/peds.2004-1073

19. Fanaroff JM, Wilson-Costello DE, Newman NS, et al. Treated hypotension is associated with neonatal morbidity and hearing loss in extremely low birth weight infants. Pediatrics 2006;117(4):1131-5. doi: 10.1542/peds.2005-1230

20. El-Khuffash AF, McNamara PJ. Neonatologist-performed functional echocardiography in the neonatal intensive care unit. Semin Fetal Neonatal Med 2011;16(1):50-60. doi: 10.1016/j.siny.2010.05.001

21. Ficial B, Finnemore AE, Cox DJ, et al. Validation study of the accuracy of echocardiographic measurements of systemic blood flow volume in newborn 
infants. $J$ Am Soc Echocardiogr 2013;26(12):1365-71. doi: 10.1016/j.echo.2013.08.019 [published Online First: 2013/10/01]

22. Sinha AK, Cane C, Kempley ST. Blood flow in the common carotid artery in term and preterm infants: reproducibility and relation to cardiac output. Arch Dis Child Fetal Neonatal Ed 2006;91(1):F31-5. doi: 10.1136/adc.2004.058172

23. Murdoch EMS, A.K; Shanmugalingam,S.T; Smith,G.C.S; Kempley,S.T. Doppler Flow Velocimetry in the Superior Mesenteric Artery on the First Day of Life in Preterm Infants and the Risk of Neonatal Necrotizing Enterocolitis. Pediatrics 2006;118(5) doi: 10.1542/peds.2006-0272

24. Jasper HH. Report of the committee on methods of clinical examination in electroencephalography. The International Federation of Clinical Neurophysiology 1957;10(2):370-75. doi: 10.1016/0013-4694(58)90053-1

25. Dunne JM, Wertheim D, Clarke P, et al. Automated electroencephalographic discontinuity in cooled newborns predicts cerebral MRI and neurodevelopmental outcome. Arch Dis Child Fetal Neonatal Ed 2017;102(1):F58-F64. doi: 10.1136/archdischild-2015-309697

26. Papile LA, Burstein J, Burstein R, et al. Incidence and evolution of subependymal and intraventricular hemorrhage: a study of infants with birth weights less than 1,500 gm. J Pediatr 1978;92(4):529-34. [published Online First: 1978/04/01]

27. Dempsey EM, Al Hazzani F, Barrington KJ. Permissive hypotension in the extremely low birthweight infant with signs of good perfusion. Arch Dis Child Fetal Neonatal Ed 2009;94(4):F241-4. doi: 10.1136/adc.2007.124263

28. Benjamini Y, Hochberg Y. Controlling the False Discovery Rate: A Practical and Powerful Approach to Multiple Testing. Journal of the Royal Statistical Society Series B (Methodological) 1995;Vol. 57(No. 1):289-300.

29. Bai-Horng Su TW, Mitsumasa Shimizu, Masayoshi Yanagisawa. Echocardiographic assessment of patent ductus arteriosus shunt flow pattern in premature infants. Archives of Disease in Childhood 1997;77:F36-F40.

30. Tsuji M, Saul JP, Plessis Ad, et al. Cerebral Intravascular Oxygenation Correlates With Mean Arterial Pressure in Critically Ill Premature Infants. Pediatrics 2000;106(4):625-32.

31. Soul JS, Hammer PE, Tsuji M, et al. Fluctuating pressure-passivity is common in the cerebral circulation of sick premature infants. Pediatr Res 2007;61(4):46773. doi: 10.1203/pdr.0b013e31803237f6 [published Online First: 2007/05/23] 
32. Batton BJ, Li L, Newman NS, et al. Feasibility study of early blood pressure management in extremely preterm infants. J Pediatr 2012;161(1):65-9 e1. doi: 10.1016/j.jpeds.2012.01.014

33. Rabe H, Diaz-Rossello JL, Duley L, et al. Effect of timing of umbilical cord clamping and other strategies to influence placental transfusion at preterm birth on maternal and infant outcomes. The Cochrane database of systematic reviews 2012(8):CD003248. doi: 10.1002/14651858.CD003248.pub3

34. Fogarty M, Osborn DA, Askie L, et al. Delayed vs early umbilical cord clamping for preterm infants: a systematic review and meta-analysis. Am J Obstet Gynecol 2018;218(1):1-18. doi: 10.1016/j.ajog.2017.10.231

\section{FIGURES}

Figure 1. Participant flow through the study.

Figure 2: Mean Arterial BP in each study arm, from continuously downloaded BP data, averaged in 1-hour periods. Statistical analysis was performed on data averaged in 4-hour epochs, by mixed effects general linear model, with Benjamini-Hochberg correction for multiple comparisons. Periods when there were significant effects of time epoch and study arm are indicated by blue/dark bars. (Values are hourly mean $+/$ SD, for patients with invasively measured BP only ( $n=51$; Active $=17$, Moderate $=18$, Permissive $=16$ ). 
